\title{
Genetics of hearing loss in the Arab population of Northern Israel
}

\author{
Nada Danial-Farran ${ }^{1,2,3} \cdot$ Zippora Brownstein $^{3} \cdot$ Suleyman Gulsuner $^{4} \cdot$ Luna Tammer $^{3} \cdot$ Morad Khayat $^{1} \cdot$ Ola Aleme $^{1}$. \\ Elena Chervinsky ${ }^{1}$ - Olfat Aboleile Zoubi ${ }^{1} \cdot$ Tom Walsh $^{4} \cdot$ Gil Ast $^{3} \cdot$ Mary-Claire King ${ }^{4} \cdot$ Karen B. Avraham $\mathbb{D}^{3}$. \\ Stavit A. Shalev ${ }^{1,2}$
}

Received: 21 February 2018 / Revised: 18 June 2018 / Accepted: 26 June 2018 / Published online: 23 August 2018

(c) The Author(s) 2018. This article is published with open access

\begin{abstract}
For multiple generations, much of the Arab population of Northern Israel has lived in communities with consanguineous marriages and large families. These communities have been particularly cooperative and informative for understanding the genetics of recessive traits. We studied the genetics of hearing loss in this population, evaluating 168 families from 46 different villages. All families were screened for founder variants by Sanger sequencing and 13 families were further evaluated by sequencing all known genes for hearing loss using our targeted gene panel HEar-Seq. Deafness in 34 of 168 families (20\%) was explained by founder variants in GJB2, SLC26A4, or OTOF. In 6 of 13 families (46\%) evaluated using HEar-Seq, deafness was explained by damaging alleles of SLC26A4, MYO15A, OTOG, LOXHD1, and TBC1D24. In some genes critical to hearing, it is particularly difficult to interpret variants that might affect splicing, because the genes are not expressed in accessible tissue. To address this problem for possible splice-altering variants of MYO15A, we evaluated minigenes transfected into HEK293 cells. Results revealed exon skipping in the message of MYO15A c.9083+6T $>$ A, and intron retention in the message of $M Y O 15 \mathrm{~A} \mathrm{c} .8340 \mathrm{G}>\mathrm{A}$, in each case leading to a premature stop and consistent with cosegregation of homozygosity for each variant with hearing loss. The profile of genetics of hearing loss in this population reflects the genetic heterogeneity of hearing loss and the usefulness of synthetic technologies to evaluate potentially causal variants in genes not expressed in accessible tissues.
\end{abstract}

These authors contributed equally: Karen B. Avraham, Stavit A. Shalev.

Electronic supplementary material The online version of this article (https://doi.org/10.1038/s41431-018-0218-z) contains supplementary material, which is available to authorized users.

Karen B. Avraham

karena@tauex.tau.ac.il

$\triangle$ Stavit A. Shalev

stavit_sh@clalit.org.il

1 Genetics Institute, Emek Medical Center, Afula, Israel

2 Rappaport Faculty of Medicine, Technion-Israel Institute of Technology, Haifa, Israel

3 Department of Human Molecular Genetics and Biochemistry, Sackler Faculty of Medicine and Sagol School of Neuroscience, Tel Aviv University, Tel Aviv, Israel

4 Department of Medicine, Division of Medical Genetics, University of Washington, Seattle, WA, USA

\section{Introduction}

The identification of alleles causing deafness has advanced dramatically in the past decade as a result of the availability of technologies such as next-generation sequencing (NGS) $[1,2]$. Applying the NGS technology to families with high consanguinity, living in isolated populations, holds promising potential for characterizing rare alleles causing recessive phenotypes, such as hereditary hearing loss. We focused on the Arab population from northern Israel, the majority of whom live in villages that were founded by a few individuals roughly ten generations ago [3] (Fig. 1). These communities are relatively isolated due to the preference for consanguineous marriages. Occasionally, a rare variant may expand among the descendants of the carriers through a founder effect mechanism and become prevalent in specific villages. Deciphering the dispersion of variants in these communities [4] facilitates relevant and precise genetic counseling for the families. Moreover, acknowledging the effect of genetic variation on a hearing disability might be the cornerstone of future research directed toward restoring hearing. 


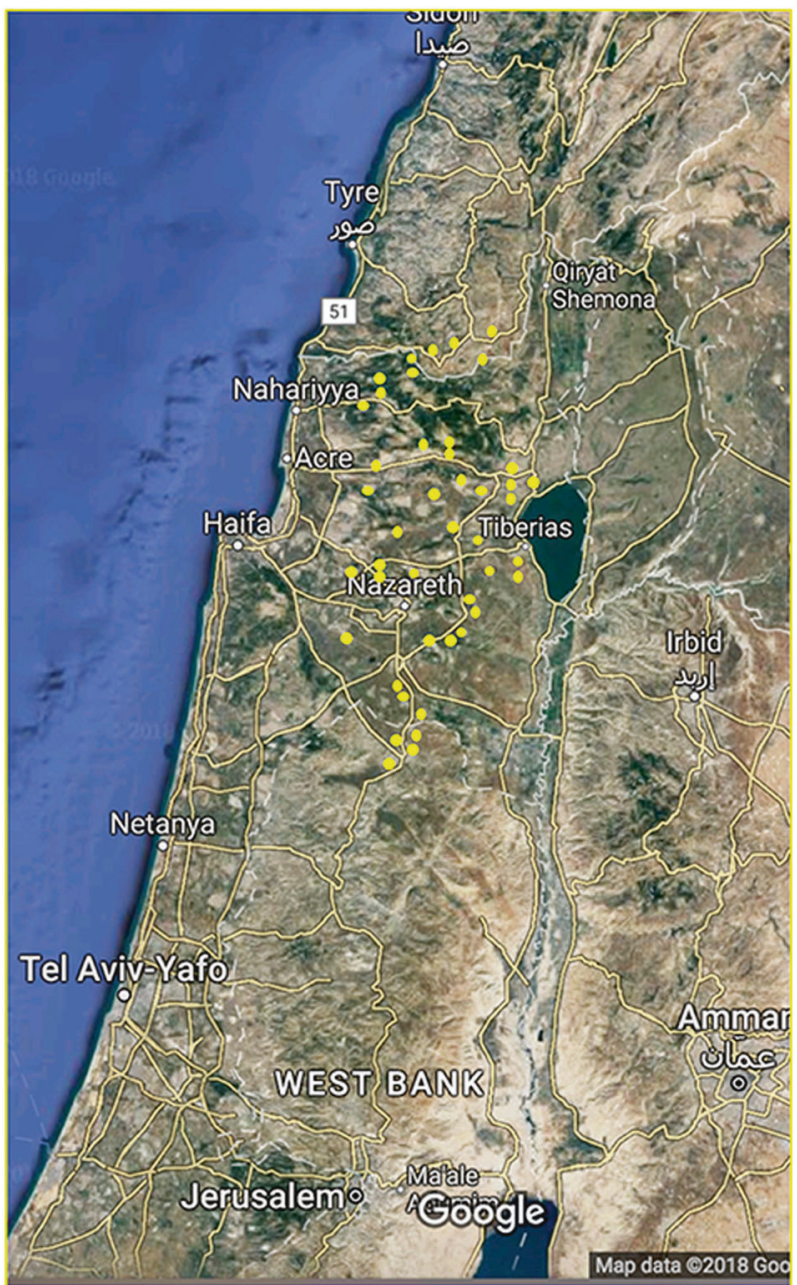

Fig. 1 Northern Israel, with home villages of the participants shown in yellow. Reproduced with permission from Google, Mapa GISrael, ORION-ME. (https://www.google.com/maps/@32.6499621,35.4502344, $224900 \mathrm{~m} /$ data $=! 3 \mathrm{~m} 1 ! 1 \mathrm{e} 3 \mathrm{hl}=\mathrm{en})$

Our cohort was comprised of 168 Arab families with nonsyndromic hearing loss (NSHL). The members of the population live in 46 different villages in the north of Israel, with long traditions of consanguineous marriage and a long history of endogamy [5]. In this study, we used NGS technology, which led to the identification of novel alleles in SLC26A4, MYO15A, OTOG, LOXHD1, and TBCID24 that are associated with deafness. In addition, we also examined the consequences of two splicing variants in the MYO15A gene that we identified in these families.

\section{Subjects and methods}

\section{Families}

This study was approved by the Ethical Committees of the Emek Medical Center and Tel Aviv University, the
Helsinki Committee of the Israel Ministry of Health, and by the Human Subjects Division of the University of Washington. A medical history was obtained from all participating individuals, including a detailed pedigree emphasizing hearing loss and other relevant symptoms. Of the 168 families, 151 reported consanguinity. Affected individuals were asked for details about the severity of hearing loss, age of onset, symmetry of hearing loss, use of hearing aids or cochlear implants, experience of tinnitus, exposure to ototoxic drugs and/or noise, pathological conditions of the inner ear, and vestibular function. After acquiring signed informed consent, we obtained fresh blood samples for DNA extraction.

\section{DNA sequencing}

DNA extraction was performed with the DNeasy Blood \& Tissue Kit (Qiagen, Hilden, Germany). First, DNA from all families was sequenced for $G J B 2$ by Sanger sequencing, using the BigDye Terminator Cycle Sequencing Kit (Applied Biosystems, Foster City, CA, USA), and analyzed using the ABI PRISM 3100 Genetic Analyzer (Applied Biosystems) according to the manufacturer's instructions. We then applied massively parallel sequencing (MPS) of deafness genes using the HEar-Seq4 panel, modified from Hear-Seq2 [6]. Briefly, 375 protein-coding genes and microRNAs (miRNAs) were captured using the Agilent SureSelect Target Enrichment System (Agilent, Santa Clara, CA, USA). The list of genes and miRNAs appears in Supplementary Table S1, indicating source of gene, mode of inheritance and association to syndromes. The UCSC Genome Browser hg19 coordinates of the genes were submitted to eArray (Agilent Technologies) in order to design cRNA oligonucleotides to cover all exons and $10 \mathrm{bp}$ of flanking introns. The choice of genes was based on their association with deafness in humans and mice or literature evidence for their expression in the cochlea (Supplementary Table S1). Molecular barcodes were assigned, and 48 samples were multiplexed and sequenced in a single flowcell of the Illumina HiSeq with 100 bp paired-end reads. MPS was performed at the Technion High-Throughput Sequencing Center (Haifa, Israel) and the King laboratory. Results were validated by Sanger sequencing and checked for cosegregation with hearing loss in the families. The prevalence of allele frequencies was examined in the hearing-impaired population and compared to that in the relevant ethnic population. Variants were uploaded to the LOVD locusspecific database (www.lovd.nl/) and ClinVar (https://www. ncbi.nlm.nih.gov/clinvar/).

\section{In silico validation of splice variants}

Six prediction tools were used to estimate the splice site strength of different sequences. Five of these, 
SpliceSiteFinder, GeneSplicer, Splice Site Prediction by Neural Network, MaxEntScan, and Human Splicing Finder are available through Alamut software v.2.3 (http://www. interactive-biosoftware.com). The sixth is the Analyzer Splice Tool (http://host-ibis2.tau.ac.il/ssat/SpliceSiteFrame.htm).

\section{Splicing assays}

Two pairs of minigene clones were designed for the MYO15A variants: c. $8340 \mathrm{G}>\mathrm{A}$, and c. $9083+6 \mathrm{~T}>\mathrm{A}$. Each pair included one plasmid with the wild-type sequence, and a second plasmid with the mutant sequence. For c. $8340 \mathrm{G}>\mathrm{A}$, the insert was amplified from exon 45 downstream to exon 47 (chr17:g.18,154,705-18,155,383), and for c. $9083+6 \mathrm{~T}>\mathrm{A}$ from exon 51 downstream to exon 53 (chr17:g.18,157,808-18,158,983). PCR amplification from DNA of wild-type and homozygote family members was carried out using the primers: 5'-GCTGCCAACGGAGC CAGG-3' and 5'-CAGAACCTTGGAGGACATGC-3'; 5'CCTGCCTTCGCATCTCTG- $3^{\prime}$ and 5'-TGACCATCCT CAGGAGTTTGA-3'; respectively. A PCR reaction was designed to open the pcDNA ${ }^{\mathrm{TM}} 5 / \mathrm{FRT}$ plasmid using KAPA HiFi HotStart ReadyMixPCR Kit (Kapa Biosystems, Wilmington, DE, USA), with the primers: pcDNA5-FRT-Fw: GGCGGCCGCTCGAGTCTA, and pcDNA5-FRT-Rv: CGCTAGAGTCCGGAGGCT, length: $5062 \mathrm{bp}$.

Flp-In ${ }^{\mathrm{TM}}-293$ cell lines (Thermo Fisher Scientific, Waltham, MA, USA) were cultured in Dulbecco's Modified Eagle's Medium (DMEM) supplemented with $4.5 \mathrm{~g} / \mathrm{ml}$ glucose (Biological Industries, Beit Haemek, Israel), 10\% Fetal Calf Serum (FCS), $0.29 \mathrm{mg} / \mathrm{ml} \mathrm{L-glutamine,} 100 \mathrm{U} / \mathrm{ml}$ penicillin, $0.1 \mathrm{mg} / \mathrm{ml}$ streptomycin, and $1 \mathrm{U} / \mathrm{ml}$ nystatin (Biological Industries). Cells were grown in $100 \mathrm{~mm}$ culture dishes under standard conditions at $37^{\circ} \mathrm{C}$ in a humidified atmosphere with $5 \% \mathrm{CO}_{2}$.

For the minigene assay, $\sim 250 \mathrm{~K}$ cells were seeded in 100 $\mathrm{mm}$ culture dishes. Twenty-four hours later, the cells were transfected using TransIT ${ }^{\circledR}$-LT1 transfection reagent (Mirus Bio, Madison, WI, USA), according to the manufacturer's protocol. After $48 \mathrm{~h}$, RNA was extracted from the cells.

For $M Y O 15 \mathrm{~A}$ c. $8340 \mathrm{G}>\mathrm{A}$, stable cell lines were developed by co-transfection of $\sim 250 \mathrm{~K}$ Flp-In ${ }^{\mathrm{TM}}-293$ cells seeded in $100 \mathrm{~mm}$ culture dishes, with the $\mathrm{pcDNA}^{\mathrm{TM}} 5 / \mathrm{FRT} /$ TO expression construct and the pOG44 Flp-Recombinase Expression Vector, in a ratio of 1:9. Forty-eight hours later, the cells were harvested and transferred to $15 \mathrm{~cm}$ culture dishes. Stable transfectants were grown for three weeks and selected with Hygromycin B antibiotics.

\section{RNA isolation and RT-PCR}

Total RNA extraction was performed using TRI Reagent (Merck, Darmstadt, Germany). For RT-PCR reactions, $2 \mu \mathrm{g}$ of total RNA was amplified by reverse transcriptase (RT) in a final volume of $20 \mu \mathrm{l}$ containing: $5 \mathrm{mM}$ DTT, $0.5 \mathrm{mM}$ dNTPs, $2.5 \mu \mathrm{M}$ oligo d(T) primer, $2 \mathrm{U}$ of SuperScript ${ }^{\circledR}$ III Reverse Transcriptase (Thermo Fisher Scientific), and RT buffer. The final mixture was incubated for $1 \mathrm{~h}$ at $50^{\circ} \mathrm{C}$, and then $5 \mathrm{~min}$ at $85^{\circ} \mathrm{C}$ to inactivate the enzymes.

To specifically amplify the spliced cDNA products derived from the expressed minigene, rather than the endogenous gene, we designed a pair of primers: a forward pcDNA $^{\mathrm{TM} 5 / F R T / T O-s p e c i f i c ~ p r i m e r ~ a n d ~ a ~ r e v e r s e ~ p r i m e r ~}$ specific to the inserted minigene $3^{\prime}$ sequence. Amplification was performed for 30 cycles, $94^{\circ} \mathrm{C}$ for $30 \mathrm{~s}, 60^{\circ} \mathrm{C}$ for $45 \mathrm{~s}$ and $72^{\circ} \mathrm{C}$ for $1 \mathrm{~min}$. The products were confirmed by Sanger sequencing.

\section{Results}

\section{Sanger sequencing screen for known variants}

Affected probands were screened by Sanger sequencing, for the coding sequence in $G J B 2$, and for one prevalent variant in SLC26A4. This screen resolved 34 cases out of 168 . The variants detected in GJB2 (NM_004004.5) were: c.35delG $(18 / 34), \quad$ c. $229 \mathrm{~T}>\mathrm{C} \quad(3 / 34), \quad$ c. $-23+1 \mathrm{G}>\mathrm{A} \quad(2 / 34)$, c.301_303delGAG (1/34), and c.167delT (1/34), all in the homozygous state. Two additional compound heterozygotes were detected by the initial screening, GJB2 c.[35delG]; [109G>A] and c.[35delG];[298C >T] (2/34). Moreover, we screened for the SLC26A4 c.1197delT variant, as it was previously found in the northern Israel Arab population [7] and identified seven deaf individuals who were homozygous for this variant. Overall, the initial screen yielded a $20.2 \%(34 / 168)$ detection rate.

\section{Targeted deafness gene panel (MPS)}

Thirteen unsolved families with congenital NSHL were analyzed by high-throughput sequencing with our targeted deafness panel, HEar-Seq (Supplementary Table S1) [6]. All families presented a recessive mode of inheritance with non-syndromic, moderate to severe or profound hearing loss, with congenital or prelingual onset (Table 1). The analysis of NGS data led to the identification of five novel variants in the known deafness genes MYO15A, LOXHD1, TBC1D24, and OTOG, all of which were associated with pathogenicity, and categorized according to the ACMG guidelines [4] (Table 1). HGVS nomenclature and LOVD IDs are presented in Supplementary Table S2. An additional known disease-causing variant was identified in the SLC26A4 gene (Table 1). All variants segregated with hearing loss in the families (Fig. 2). As part of the validation interpretation, we screened for all the identified variants in a 


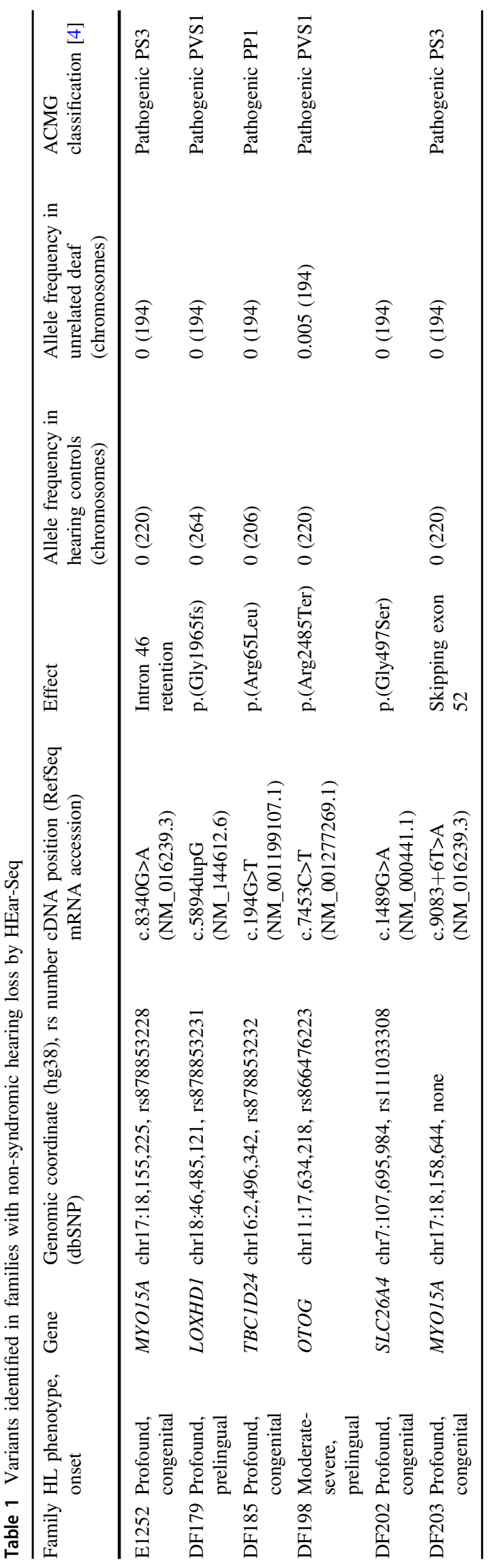

population comprised of hearing controls, sharing the same ethnicity. None of the identified variants were present in any of the chromosome samples. Subsequently, we sequenced the identified variants across 120 undiagnosed hearing impaired probands. A diagnosis for one family revealed that two deaf siblings from the same village as the DF198 family are homozygous for OTOG c.7453C $>\mathrm{T}$, p. (Arg2485Ter).

\section{Validation of splicing variants}

We further investigated the effects of two variants in MYO15A: c. $9083+6 \mathrm{~T}>\mathrm{A}$, exon 52 , and c. $8340 \mathrm{G}>\mathrm{A}$, p. (Thr2780Thr), in the last nucleotide of exon 46, as we hypothesized that they may affect splicing. Because the tissues that express this gene are inaccessible, we could not obtain RNA from the patients in order to evaluate the effect of the variants on expression directly. For c. 9083 $+6 \mathrm{~T}>\mathrm{A}$, a transient minigene experiment revealed a differential expression pattern between the wild type and mutated plasmid. This was then examined by RT-PCR after extraction of RNA. The results revealed that there are two isoforms that are expressed normally: one with full exon inclusion, and the second without exon 52 (Fig. 3a). In contrast, the mutant sequence expressed only the second isoform without exon 52 .

The minigene assay failed to express the insert with c. $8430 \mathrm{G}>\mathrm{A}$ using a transient vector. For this reason, we used the Flp-In ${ }^{\mathrm{TM}}-\mathrm{System}$ to generate two stably transfected 293 cell lines, one with the wild type and the second with the mutant. It is important to note that the two minigenes were inserted into the same genomic locus and in the same orientation. The splicing pattern was then examined by RT-PCR after extraction of RNA. Genomic DNA was extracted from each cell line in order to confirm that the integrated minigene did not acquire any unforeseen variant. In the wild-type stable cell line, the RT-PCR results included two mRNA products: the full exon inclusion isoform, and a second isoform with retention of intron 46. In the mutated cell line, the full exon inclusion isoform was absent in all examined colonies $(n=5)$, and only the isoform with intron retention was detected $(n=$ 5) (Fig. 3e-g). Importantly, the $\mathrm{G}>\mathrm{A}$ variant in the last nucleotide of exon 46 eliminates the full exon inclusion isoform, indicating that this variant impairs splicing of exon 46.

\section{Discussion}

The objective of the current study was to identify genetic causes of hearing impairment in consanguineous families living in northern Israel. The detection rate of alleles 
a

E1252

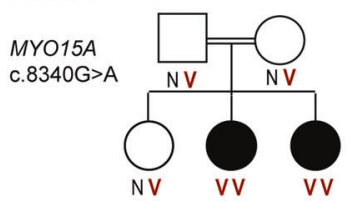

d

DF198

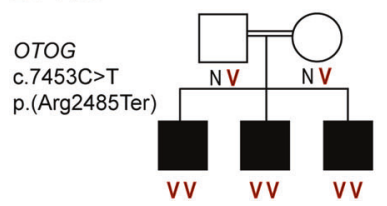

b

DF179

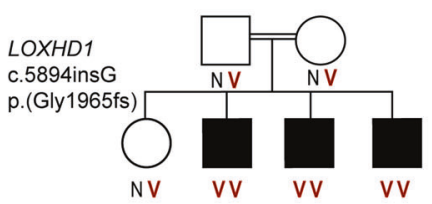

e
DF202

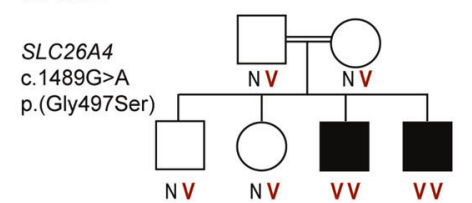

DF185

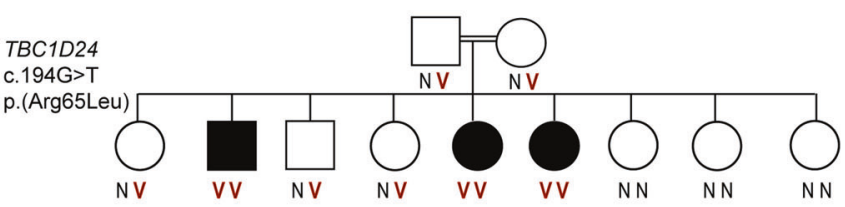

f
Fig. 2 Pedigrees of the families with NSHL investigated by the HEarSeq deafness panel and the segregation analysis. a Family E1252. b
Family DF179. c Family DF185. d Family DF198. e Family DF202. f Family DF203. N, wild type; V, variant affecting function in our cohort by high-throughput sequencing was $47 \%$ (6/13), and included novel alleles associated with hearing loss. This outcome is in agreement with other studies conducted in various endogamous as opposed to non-consanguineous populations worldwide [8-10]. Our study highlights the advantages of studying isolated populations for detecting rare recessive variants associated with hearing impairment. Most of the Arab population in Israel live in villages and towns that were founded by few founders about ten generations ago and is characterized by frequent consanguineous/endogamous marriages and a high fertility rate [11]. Each of the localities may therefore be regarded as a small isolate. According to their geographic distribution, the genetic diseases may be classified into diseases that are relatively frequent in all the population, such as thalassemia, usually caused by ancient variants. Diseases that are frequent in more than one community, and those which are confined to a local community, were found to be caused by variants that are either relatively recent or were introduced recently in the population, most of them in a homozygous state. Therefore, it is probable that most of the genetic diseases in this population is due to founder effect.

All the variants detected in our cohort are recessive and the hearing-impaired individuals represent a homozygous state. The novel variant, c.5894dupG in the LOXHD1 gene, is a frameshift variant that is predicted to damage the sequence downstream of position 1695, a genomic location that is evolutionarily conserved. This variant was found in three affected siblings in a homozygous state and was absent in our hearing and hearing-impaired cohorts. The variant in family DF198, c. $7453 \mathrm{C}>\mathrm{T}$, in the $O T O G$ gene, is a nonsense variant. Three affected siblings had this variant in a homozygous state. Subsequently, one deaf individual from our hearing-impairment cohort was found to be a homozygote, and then, his deaf sibling was also tested and found to be homozygous for this variant. Of note, these two individuals excluded familial relations with family DF198, although they do live in the same village. Hence, although the haplotypes were not investigated, we assume that they are from a common founder.

The variant c.194G $>$ T, p.(Arg65Leu) in TBC1D24 that was detected in family DF185, was found in three homozygous siblings, and segregation was supported by testing additional eight hearing family members. We did not find this variant in either normal or affected cohorts matched for the same ethnic background. A variant located five amino acids downstream, p.(Asp70Tyr), was previously detected in a consanguineous family with profound NSHL, as in family DF185 [12]. Of note, some variants in TBC1D24 are associated with deafness, onychodystrophy, osteodystrophy, mental retardation and seizures (DOORS). The affected members of family DF185 did not exhibit any symptoms other than deafness, as determined by detailed medical examinations. Superimposing the human variants on the crystal structure of the Drosophila ortholog defined the putative function of some of these residues, based on their location or binding with $\mathrm{PI}(4,5) \mathrm{P} 2$ [13].

The additional novel variants in $M Y O 15 A$ were detected in two families; and neither were present in our hearing or affected cohorts. Further investigation revealed that these variants are located in critical splicing regions, with strong evidence for associated pathogenicity, according to the location and to in silico analysis (Table 2). Splicing variants might alter normal splicing and result in non-functional gene products; however, different splicing patterns might actually produce different products that have a benign or even favorable effect [14]. Previous studies have shown that exonic and intronic variants that were considered to be variants with unknown significance were subsequently 


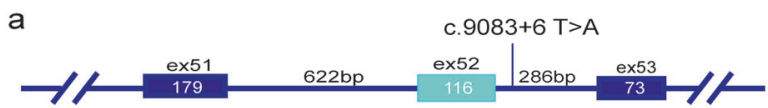

$\mathrm{b}$

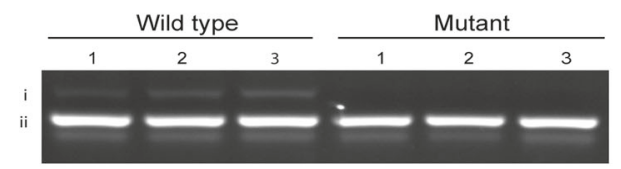

c
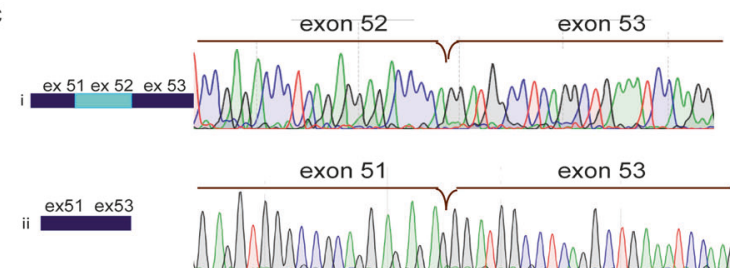

d Predicted protein sequence with exon 52 skipping

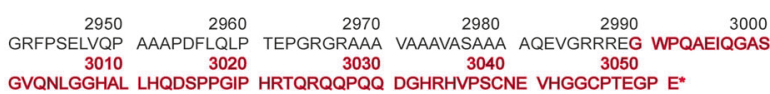

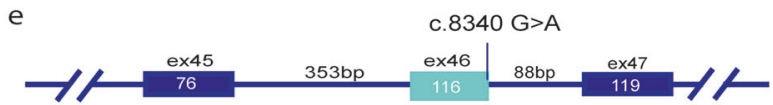

f

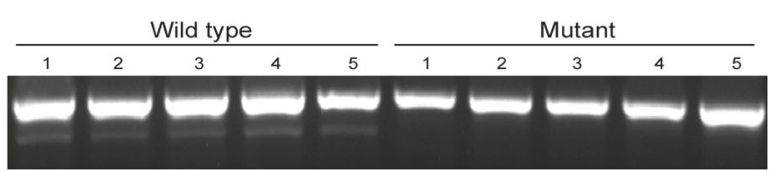

g
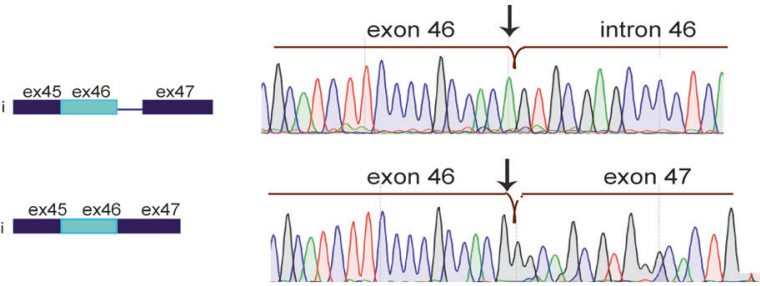

h Predicted protein sequence with intron 46 retention

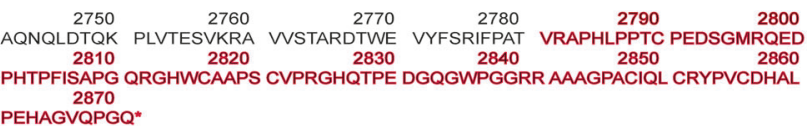
PEHAGVQPGQ*

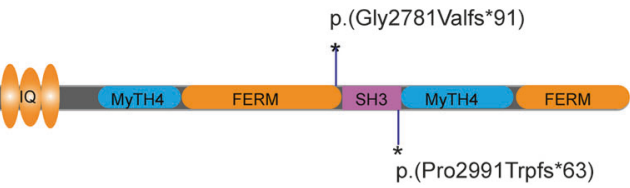

Fig. 3 Analysis of the expression pattern of the wild-type and mutant alleles of MYO15A. a, e A schematic representation of the constructs designed for minigene (for c.9083+6T $>$ A) and stable cell line (for c. $8340 \mathrm{G}>\mathrm{A}$ ) experiments, respectively. For each experiment two plasmids were prepared, one with the wild-type sequence and second with the investigated variant. b, f Agarose gel electrophoresis of cDNA

implicated in diseases by studying their effect on splicing [15]. Therefore, splicing analysis on the RNA level is a relevant starting point to determine the potential pathogenicity of a variant. Unfortunately, the evaluation of splicing variants can be limited by the lack of RNA samples, as is the case for inner ear-specific genes that are not expressed in accessible tissues. In such cases, our minigene experiments with transient vectors are an attractive alternative that can be used to investigate the effect of splicing variants on translation. Studies have shown $100 \%$ concordance when comparing the effect of splicing variants on RNA and minigene constructs $[16,17]$. This approach has been used to study other variants implicated in hearing loss, including for MYO15A, STRC, TJP2 and USH2A [18, 19], The minigene experiment described here was designed to evaluate the expression pattern of c.9083+6T $>$ A and revealed a differential splicing pattern between the wild type and the mutant alleles. Expression of the wild-type sequence was detected in the cell lines transfected with the wild-type minigene plasmid, but not with the mutated sequence. This was confirmed by sequencing. The minigene results provide evidence of the aberrant splicing pattern, which may be
PCR products prepared from RNA extracted from transfected cell lines. c, $\mathbf{g}$ A sequence chromatogram of each isoform detected by gel electrophoresis. $\mathbf{d}, \mathbf{h}$ The predicted amino acid sequence of the protein that is truncated as a result of alternative splicing. i Schematic representation of the myosin XVA protein [22] shows the location of each predicted truncation due to the splice variants

caused by disruption of U1 snRNP binding to the $5^{\prime}$ splice site that prevents splicing initiation [13] and leads to skipping exon 52.

It is important to appreciate that minigene experiments may not always be an appropriate approach to studying splicing variants. This was illustrated by the case of family E1252 (Table 1), where we investigated the consequences of the novel splicing variant c.8340G $>\mathrm{A}$ in the MYO15A gene, located in the last base pair of exon 46. In this case, the minigene technique failed to resolve the expression pattern of this genomic region, possibly because of the high GC content, and short length ( $88 \mathrm{bp}$ ) of the intron. Further analysis, using a stable cell line, successfully confirmed the altered splicing pattern. Expression of an isoform with intron retention was seen in the mutated cells but there was no full exon inclusion isoform with exon 46 . The results were confirmed by sequencing.

We can conclude from the expression patterns of both c. $8340 \mathrm{G}>\mathrm{A}$ and c. $9083+6 \mathrm{~T}>\mathrm{A}$ that the altered sequences result in aberrant proteins and that this is responsible for the lack of normal mRNA isoforms in both tested families. We predict that a defective reading frame results in a truncated 
Table 2 Prediction tools to evaluate the donor splicing site sequence strength

\begin{tabular}{|c|c|c|c|c|c|c|}
\hline & $\begin{array}{l}\text { Splice site } \\
\text { finder }(0-100)\end{array}$ & $\begin{array}{l}\text { Max Ent } \\
\text { scan }(0-12)\end{array}$ & $\begin{array}{l}\text { NNSPLICE } \\
(0-1)\end{array}$ & $\begin{array}{l}\text { Gene splicer } \\
(0-15)\end{array}$ & $\begin{array}{l}\text { Human splice } \\
\text { finder }(0-100)\end{array}$ & $\begin{array}{l}\text { Analyzer splice } \\
\text { tool }(0-100)\end{array}$ \\
\hline $\begin{array}{l}\text { MYO15A, c. } 8340(\mathrm{G}) \\
\text { Ref. }\end{array}$ & 70.03 & 7.2 & 0.61 & 9.39 & 82 & 69.54 \\
\hline MYO15A, c.8340 (A) Alt. & NI & 0.23 & NI & 5.19 & 71.42 & 57.63 \\
\hline $\begin{array}{l}\text { Decrease in splicing } \\
\text { strength }(\%)\end{array}$ & - & $58 \%$ & - & $28 \%$ & $10.58 \%$ & $11.91 \%$ \\
\hline $\begin{array}{l}\text { MYO15A, c. } 9083+6(\mathrm{~T}) \\
\text { Ref. }\end{array}$ & 74.59 & 8.3 & 0.9 & 9.3 & 83.5 & 73.91 \\
\hline MYO15A, c.9083 (A) Atl. & NI & 3.7 & NI & 6 & 81.5 & 68.64 \\
\hline $\begin{array}{l}\text { Decrease in splicing } \\
\text { strength }(\%)\end{array}$ & - & $38.3 \%$ & - & $22 \%$ & $2 \%$ & $5.27 \%$ \\
\hline
\end{tabular}

NI not identified, WT Wild type

non-functional protein lacking the tail that contains three critical domains: $\mathrm{SH} 3, \mathrm{MyTH} 4$, and FERM, a region that is crucial for normal hearing (Fig. 3d, h, i) [20, 21].

In summary, diagnosing the genetic basis of deafness is a first crucial step in understanding the underlying pathological mechanisms causing hearing impairment, and might contribute to our knowledge of the physiological process of hearing. From a clinical perspective, our findings have dramatically improved the clinical management of patients and can be used to guide the genetic counseling provided to affected individuals and their family members. Finally, investigating the consequences of a novel variant may be the cornerstone to developing future gene-specific therapies.

Acknowledgements We wish to thank the families for their contribution to the research and to Lara Kamal for her help in preparing libraries for NGS. This work was supported by the National Institutes of Health/NIDCD R01-DC011835 (KBA, MCK). KBA is an incumbent of the Drs. Sarah and Felix Dumont Chair for Research of Hearing Disorders. This work was performed in partial fulfillment of the requirements for a Ph.D. degree by Nada Daniel-Faran (Technion).

Author contributions NDF, ZB, TW, MCK, KBA and SAS conceived the project and together with SG and LT designed and performed experiments and analyzed the data. MK, OA, EC, OAZ and SAS contributed to patient acquisition. NDF, KBA and SAS wrote the manuscript. KBA and SAS supervised the project. All authors contributed to revising and approved the manuscript for publication.

\section{Compliance with ethical standards}

Conflict of interest The authors declare that they have no conflict of interest.

Open Access This article is licensed under a Creative Commons Attribution 4.0 International License, which permits use, sharing, adaptation, distribution and reproduction in any medium or format, as long as you give appropriate credit to the original author(s) and the source, provide a link to the Creative Commons license, and indicate if changes were made. The images or other third party material in this article are included in the article's Creative Commons license, unless indicated otherwise in a credit line to the material. If material is not included in the article's Creative Commons license and your intended use is not permitted by statutory regulation or exceeds the permitted use, you will need to obtain permission directly from the copyright holder. To view a copy of this license, visit http://creativecommons. org/licenses/by/4.0/.

\section{References}

1. Neveling K, Feenstra I, Gilissen C, et al. A post-hoc comparison of the utility of sanger sequencing and exome sequencing for the diagnosis of heterogeneous diseases. Hum Mutat. 2013;34: 1721-6.

2. Metzker ML. Sequencing technologies-the next generation. Nat Rev Genet. 2010;11:31-46.

3. Zlotogora J. Genetics and genomic medicine in Israel. Mol Genet Genom Med. 2014;2:85-94.

4. Richards S, Aziz N, Bale S, et al. Standards and guidelines for the interpretation of sequence variants: a joint consensus recommendation of the American College of Medical Genetics and Genomics and the Association for Molecular Pathology. Genet Med. 2015;17:405-24.

5. Zlotogora J, Barges S, Bisharat B, et al. Genetic disorders among Palestinian Arabs. Genetic clinics in the community. Am J Med Genet A. 2006;140:1644-6.

6. Brownstein Z, Friedman LM, Shahin H, et al. Targeted genomic capture and massively parallel sequencing to identify genes for hereditary hearing loss in Middle Eastern families. Genome Biol. 2011;12:R89.

7. Everett LA, Glaser B, Beck JC, et al. Pendred syndrome is caused by mutations in a putative sulphate transporter gene $(P D S)$. Nat Genet. 1997;17:411-22.

8. Sloan-Heggen CM, Babanejad M, Beheshtian M, et al. Characterising the spectrum of autosomal recessive hereditary hearing loss in Iran. J Med Genet. 2015;52:823-9.

9. Sanyelbhaa H, Kabel A, Abo El-Naga HAE, et al. The risk ratio for development of hereditary sensorineural hearing loss in consanguineous marriage offspring. Int $\mathrm{J}$ Pediatr Otorhinolaryngol. 2017;101:7-10.

10. Zazo Seco C, Wesdorp M, Feenstra I, et al. The diagnostic yield of whole-exome sequencing targeting a gene panel for hearing impairment in The Netherlands. Eur $\mathrm{J}$ Hum Genet. 2017;25:308-14.

11. Zlotogora J. Molecular basis of autosomal recessive diseases among the Palestinian Arabs. Am J Med Genet. 2002;109:176-82. 
12. Rehman AU, Santos-Cortez RL, Morell RJ, et al. Mutations in $T B C 1 D 24$, a gene associated with epilepsy, also cause nonsyndromic deafness DFNB86. Am J Hum Genet. 2014;94:144-52.

13. Fischer B, Luthy K, Paesmans J, et al. Skywalker-TBC1D24 has a lipid-binding pocket mutated in epilepsy and required for synaptic function. Nat Struct Mol Biol. 2016;23:965-73.

14. Keren H, Lev-Maor G, Ast G. Alternative splicing and evolution: diversification, exon definition and function. Nat Rev Genet. 2010;11:345-55.

15. Pagani F, Baralle FE. Genomic variants in exons and introns: identifying the splicing spoilers. Nat Rev Genet. 2004;5:389-96.

16. Steffensen AY, Dandanell M, Jønson L, et al. Functional characterization of $B R C A 1$ gene variants by mini-gene splicing assay. Eur J Hum Genet. 2014;22:1362-8.

17. Matesanz F, Fedetz M, Barrionuevo C, et al. A splice variant in the ACSL5 gene relates migraine with fatty acid activation in mitochondria. Eur J Hum Genet. 2016;24:1572-7.
18. Baux D, Vaché C, Blanchet $\mathrm{C}$, et al. Combined genetic approaches yield a $48 \%$ diagnostic rate in a large cohort of French hearing-impaired patients. Sci Rep. 2017;7:16783.

19. Shafique S, Siddiqi S, Schraders M, et al. Genetic spectrum of autosomal recessive non-syndromic hearing loss in Pakistani families. PLoS One. 2014;9:e100146.

20. Anderson DW, Probst FJ, Belyantseva IA, et al. The motor and tail regions of myosin $\mathrm{xv}$ are critical for normal structure and function of auditory and vestibular hair cells. Hum Mol Genet. 2000;9:1729-38.

21. Fang Q, Indzhykulian AA, Mustapha $\mathrm{M}$ et al. The $133-\mathrm{kDa} \mathrm{N}-$ terminal domain enables myosin 15 to maintain mechanotransducing stereocilia and is essential for hearing. eLife. 2015; 4:e08627.

22. The UniProt Consortium. UniProt: the universal protein knowledgebase. Nucleic Acids Res. 2017;45:D158-69. 